\title{
STURMIAN THEOREMS FOR SECOND ORDER SYSTEMS
}

\author{
W. ALLEGRETTO
}

\begin{abstract}
Sturmian theorem are established for weakly coupled elliptic systems generated in a bounded domain by the expressions $l_{1} \vec{u}=-\Delta \vec{u}+A \vec{u}, l_{2} \vec{w}=-\Delta \vec{w}+$ $B \vec{u}$, and Dirichlet boundary conditions. Here $\Delta$ denotes the Laplace operator, and $A, B$ are $m \times m$ matrices. We do not assume that $A, B$ are symmetric, but instead essentially require $B$ irreducible and $b_{i j} \leqslant 0$ if $i \neq j$. Estimates on the real eigenvalue of $l_{2}$ with a positive eigenvector are then obtained as applications. Our results are motivated by recent theorems for ordinary differential equations established by Ahmad, Lazer and Dannan.
\end{abstract}

Let $G \subset R^{n}, n \geqslant 2$, denote a bounded domain. We consider in $H^{1.2}(G)$ the elliptic operators

$$
\begin{aligned}
& l_{1} \vec{u}=-\Delta \vec{u}+A \vec{u}, \\
& l_{2} \vec{w}=-\Delta \vec{w}+B \vec{w},
\end{aligned}
$$

where $A=\left(a_{i j}\right), B=\left(b_{i j}\right)$ are $m \times m$ matrices, $\vec{u}=\left(u_{1}, \ldots, u_{m}\right)^{\mathrm{T}}, \vec{w}=\left(w_{1}, \ldots, w_{m}\right)^{\mathrm{T}}$ are $m$ vectors, $\Delta$ is the Laplace operator, and $H^{1,2}(G)$ represents the usual Sobolev space with the norm

$$
\|\vec{u}\|_{1,2}^{2}=\sum_{1}^{m}\left\|u_{i}\right\|_{1,2}^{2}=\sum_{i=1}^{m} \int \sum_{|\alpha| \leqslant 1}\left|D^{\alpha} u_{i}\right|^{2} .
$$

We do not assume that $A, B$ are symmetric.

If $m=1$ the typical Sturmian theorem states that every solution of $l_{2} \vec{w}=\overrightarrow{0}$ must vanish somewhere in $G$ (or $\bar{G}$ ) if $A, B$ are suitably related and there exists a nontrivial solution $\vec{u} \geqslant \overrightarrow{0}$ of $l_{1} \vec{u} \leqslant \overrightarrow{0}$ in $H_{0}^{1,2}(G)$. Precise formulations of this and related results, together with the needed regularity hypotheses, may be found in the books by Swanson [12] and Kreith [9] and the more recent survey articles [13, 14]. Such results can, in particular, be used for eigenvalue comparison, and the above references contain several examples of such instances.

It is the purpose of this paper to establish a Sturmian theorem and consequent eigenvalue comparison for the weakly coupled systems given by (1), (2). Unlike many earlier results, we do not deal with determinants of prepared matrix solutions or with $h$-oscillatory vector solutions (see the above references for clarification of these concepts). Instead, our results deal with the sign of the components of the solutions

Received by the editors June 12, 1984 and, in revised form, August 14, 1984.

1980 Mathematics Subject Classification. Primary 35B05, 35P15, $35 \mathrm{~J} 55$.

Key words and phrases. Sturmian theorem, elliptic system, eigenvalue, positive operator. 
of (1),(2) and are closer in formulation to the theorems established for ordinary differential systems by Ahmad and Lazer [1, 2] and, more recently, Dannan [5]. Indeed, it appears that our results, even when specialized to the case $n=1$, actually give new criteria not contained in the theorems in $[1,2,5]$.

Finally we recall that Protter has developed a general method for establishing Sturmian theorems and eigenvalue comparison for single equations and systems; see, in particular, [11]. His method depends on the introduction of arbitrary vector fields which are then to be strategically chosen. Many-we conjecture all-of the known scalar theorems can be obtained in this way. This approach is alson very relevant here, as we indicate below.

We now state our assumptions on the coefficients. We assume that $a_{i j}, b_{i j} \in L^{q}(G)$ with $q>n$. We shall also assume for most of the paper that the condition

$$
b_{i j} \leqslant 0 \quad \text { if } i \neq j
$$

holds in $G$, and shall indicate how this may be weakened at the end. The matrix $B$ will be assumed irreducible: e.g., there exists a permutation $\sigma$ of $\{1, \ldots, m\}$ such that $b_{\sigma(i), \sigma(i+1)}, b_{\sigma(m), \sigma(1)}$, are not zero a.e. in $G$ for $i=1, \ldots, m-1$. The regularity assumptions could be weakened slightly, and $\Delta$ could be replaced by a more general second order expression without essentially changing the proofs. The weak coupling of (1), (2), however, is extensively used. Observe, finally, that the regularity results of Ladyzhenskaya and Uraltseva [10, p. 404] imply that any solution $\vec{v} \in H^{1.2}(G)$ of $l_{j} \vec{v}=\overrightarrow{0}$ in $G$ for $j=1,2$ is actually in $C^{1}(G)$. Furthermore, if $\partial G$ is smooth and $\vec{v} \in H_{0}^{1,2}(G)$ then also $\vec{v} \in C^{\alpha}(\bar{G})$ for some $\alpha>0$.

Our first result is a Picone-type identity for systems (1), (2) which is related to the scalar identities given, e.g., in $[3,9,12]$, and whose proof can be easily obtained by direct calculation.

We write $\vec{u}<\vec{v}$ iff $u_{i}<v_{i}$ for $i=1, \ldots, m$. Similarily, other inequalities between vectors are understood to hold for each component.

Lemma 1. Assume $v_{i} \neq 0$ in $G, v_{i} \in C^{1}(G)$ and $\phi_{i} \in C_{0}^{1}(G)$ for $i=1, \ldots, m$. Let $B_{1}, B_{2}$ be the quadratic forms associated with $l_{1}, l_{2}$, respectively. Then

$$
\begin{gathered}
\int_{G} \sum_{k, j} v_{j}^{2}\left[D_{k}\left(\frac{\phi_{j}}{v_{j}}\right)\right]^{2}-\frac{1}{2} \sum_{i, j}\left\{b_{i j}\left(\frac{\phi_{i}^{2}}{v_{i}}\right) v_{j}+b_{j i}\left(\frac{\phi_{j}^{2}}{v_{j}}\right) v_{i}-\left[a_{i j}+a_{j i}\right] \phi_{i} \phi_{j}\right\} \\
=B_{1}(\vec{\phi}, \vec{\phi})-B_{2}(\vec{v}, \vec{\tau}),
\end{gathered}
$$

where

$$
\tau=\left\{\phi_{i}^{2} / v_{i}, i=1, \ldots, m\right\}^{\mathrm{T}}, \quad \vec{v}=\left\{v_{1}, \ldots, v_{m}\right\}^{\mathrm{T}}, \quad \vec{\phi}=\left\{\phi_{1}, \ldots, \phi_{m}\right\}^{\mathrm{T}} .
$$

Corollary 1. Assume

$$
\begin{aligned}
& \left\{\begin{array}{l}
b_{i j} b_{j i} \geqslant\left[a_{i j}+a_{j i}\right]^{2} / 4, \quad i \neq j, \\
b_{i i} \leqslant a_{i i}, \\
B_{1}(\vec{u}, \vec{u}) \leqslant 0 \quad \text { for some } \vec{u} \in H_{0}^{1}(G), \vec{u} \neq 0 .
\end{array}\right.
\end{aligned}
$$


If there exists a positive $C^{1}$ vector $\vec{v}$ in $G$ such that $l_{2} \vec{v} \geqslant \overrightarrow{0}$, then $B(\vec{u}, \vec{u})=0, l_{2} \vec{v}=\overrightarrow{0}$ and there exist nonzero constants $c_{i}$, such that $u_{i}=c_{i} v_{i}$ and $b_{i j}=\left(\left(a_{i j}+a_{j i}\right) / 2\right)\left(c_{j} / c_{i}\right)$ in $G$ for $i, j=1, \ldots, m$.

Proof. Observe that condition (3) and assumption (i) imply that the left side of (4) is nonnegative definite. Approximate $\vec{u}$ by a sequence of $C_{0}^{\infty}$ functions $\left\{\vec{\phi}_{r}\right\}$ and observe that (4) yields an immediate contradiction unless $B_{1}(\vec{u}, \vec{u})=0$. Furthermore, in the latter case, we conclude from the first term on the left side of (4) that $u_{i}=c_{i} v_{i}$, as $\operatorname{grad}\left(u_{i} / v_{i}\right) \equiv 0$ and $G$ is a domain. From the second part of the left side and assumption (i) we conclude that

$$
b_{i j} \frac{u_{i}}{v_{i}}=\frac{a_{i j}+a_{j i}}{2} \frac{u_{j}}{v_{j}}
$$

for all $i, j$. By the irreducibility assumption, $c_{i} \neq 0$ for $i=1, \ldots, m$, and the result follows.

Observe that if $B$ is symmetric then $\vec{u}=c D \vec{v}$ in the above for some $D=\operatorname{diag}( \pm 1)$ and constant $c$. If also $B=A$ then $D=I$.

COROLlARY 2. If any of the following conditions hold, then no such vector $\vec{v}$ exists:

(i) a component of $\vec{u}$ changes sign in $G$;

(ii) $l_{2} \vec{v} \not \equiv \overrightarrow{0}$ in $G$;

(iii) $B_{1}(\vec{u}, \vec{u})<0$ in $G$;

(iv) there exist $i, j$ such that $b_{i j}$ is not a constant multiple of $\left(\left(a_{i j}+a_{j i}\right) / 2\right)$;

(v) there exist $i$ such that $b_{i i}<a_{i i}$ in a set of positive measure.

As mentioned in the introduction an alternate proof of these results can be obtained by using one of Protter's theorems instead of a Picone identity. Specifically, it is possible to start by judiciously selecting a vector field and by properly collecting terms in the general matrices on p. 507 of [11]. Our choice of a direct proof was made merely for convenience.

We wish to apply the Lemma and Corollary to obtain two-sided eigenvalue estimates. We first show that suitable eigenvalues exist.

Lemma 2. $l_{2}$, with Dirichlet conditions, has a real eigenvalue $\lambda$ with a positive eigenvector.

Proof. If need be add $k I$ to $l_{2}$, with $k$ large, so that $B_{2}$ becomes coercive over $H_{0}^{1.2}(G)$. Still denoting the resulting operator by $l_{2}$, we observe that $\operatorname{Range}\left(l_{2}\right)=$ $L^{2}(G)$, by the Lax-Milgram Lemma, and $l_{2}^{-1}$ is a compact map from $L^{2}(G)$ to $L^{2}(G)$ by the Sobolev embedding theorems. Further, if $\vec{f} \geqslant \overrightarrow{0}$ and $l_{2} \vec{u}=\vec{f}$ then $B\left(\vec{u}^{-}, \vec{u}^{-}\right) \leqslant 0$ by assumption (3). Coercivity implies $\vec{u}^{-}=\overrightarrow{0}$, i.e., $l_{2}^{-1}$ leaves invariant the cone of nonnegative functions. If, further, $\vec{f} \not \overrightarrow{0}$ then $\vec{u} \not \equiv \overrightarrow{0}$ and, for each component,

$$
-\Delta u_{i}+b_{i i} u_{i} \geqslant 0, \quad u_{i}=0 \quad \text { on } \partial G .
$$

By the weak Harnack ineqality for supersolutions (see [7, p. 184]) applied to (5) we conclude that if inf $u_{i}=0$ in some ball in $G$ then $u_{i} \equiv 0$ in $G$. By the irreducibility assumption this yields the contradiction $\vec{u} \equiv \overrightarrow{0}$. Consequently, if $\vec{f} \in C_{0}^{\infty}(G), \vec{f} \geqslant \overrightarrow{0}$, $\not \equiv \overrightarrow{0}$ in $G$ then $0<l_{2}^{-1}(\vec{f}) \in C(G)$, and for some positive constant $\beta, l_{2}^{-1}(\vec{f})>\beta \vec{f}$ in 
$G$. We apply a theorem of Krasnoselskii [8, p. 67] and conclude that $l_{2}^{-1}$ has a real eigenvalue $\lambda$ with eigenvector $\vec{v} \geqslant \overrightarrow{0}$ in $G$. Consequently, the same holds for $l_{2}$ and, further, $\vec{v}>\overrightarrow{0}$ in $G, \vec{v} \in C^{1}(G)$.

Since $l_{2}^{*}$ satisfies the same conditions, we note that there is at most one such eigenvalue $\lambda$. With more assumptions, $\lambda$ can be further characterized [8], but we shall have no need of this.

Given any expression of type $-\Delta \vec{u}+C \vec{u}$ with $C=\left(c_{i j}\right)$, we shall denote by $\lambda\left(c_{i j}\right)$ the above eigenvalue. For notational convenience, we let $-\sqrt{b_{i i} b_{i i}}$ denote $b_{i i}$ in the sequel.

THEOREM 1. $\lambda\left(\left(b_{i j}+b_{j i}\right) / 2\right) \leqslant \lambda\left(b_{i j}\right) \leqslant \lambda\left(-\sqrt{b_{i j} b_{j i}}\right)$.

Proof. $\lambda\left(b_{i j}\right) \geqslant \lambda\left(\left(b_{i j}+b_{j i}\right) / 2\right)$ follows from the Courant Min-Max principle once we observe that, since $\left(B+B^{*}\right) / 2$ is symmetric, then $\lambda\left(\left(b_{i j}+b_{j i}\right) / 2\right)$ is the smallest eigenvalue, as may be seen by replacing both $a_{i j}$ and $b_{i j}$ by $\left(b_{i j}+b_{j i}\right) / 2$ in identity (4). Finally, choosing $a_{i j}=-\sqrt{b_{i j} b_{j i}}$ in (4) yields $\lambda\left(b_{i j}\right) \leqslant \lambda\left(-\sqrt{b_{i j} b_{j i}}\right)$.

Note that this result is optimal in the sense the equality holds if $b_{i j}=b_{j i}$.

Suppose next that there exists a family of nested smooth domains $\left\{G_{t}\right\}_{t \in I}$ such that $G_{t_{1}} \Subset G_{t_{2}}$ if $t_{1}>t_{2}$ and $G_{t_{0}}=\bigcup_{t>t_{0}} G_{t}, \bar{G}_{t_{0}}=\bigcap_{t<t_{0}} G_{t}$. We require that $\partial G_{t}$ be sufficiently regular so that $\bigcap_{t<t_{0}} H_{0}^{1,2}\left(G_{t}\right)=H_{0}^{1,2}\left(G_{t_{0}}\right)$; see [15] for a discussion of this.

THEOREM 2. Let $\lambda(t)$ be the distinguished eigenvalue for the Dirichlet problem for $l_{2}$ in $G_{t}$. Then $\lambda(t)$ is continuous in $t$, and if $\operatorname{diam}\left(G_{t}\right) \rightarrow 0$ as $t \rightarrow \infty$, then $\lambda(t) \rightarrow \infty$ as $t \rightarrow \infty$.

PROof. Let $t_{r} \rightarrow t_{0}$ and assume $\lambda_{r}, \vec{v}_{r}$ are the associated eigenvalue, eigenvector. Normalize $\vec{v}_{r}$ by $\left\|\vec{v}_{r}\right\|_{0}=\int_{R_{n}}|\vec{v}|^{2}=1$. From the Theorem 1 estimate we conclude that $\lambda_{r}$ is bounded. Hence, there is a subsequence, also denoted by $\lambda_{r}$, which converges to some number $\lambda_{0}$. Since $B_{2}\left(\vec{v}_{r}, \vec{v}_{r}\right)$ is bounded we may also conclude-if need be add $k I$ again to $B_{2}$ - that $\vec{v}_{r}$ is weakly convergent in $H_{0}^{1,2}\left(R_{n}\right)$ to a function $\vec{v}$. From our regularity assumption on $\partial G_{t_{0}}$ it follows that $\vec{v} \in H_{0}^{1,2}\left(G_{t_{0}}\right)$. Now let $\phi \in C_{0}^{\infty}\left(G_{t_{0}}\right)$. Then $B_{2}\left(\phi\left(\vec{v}_{r}-\vec{v}_{s}\right), \phi\left(\vec{v}_{r}-\vec{v}_{s}\right)\right)$ is Cauchy, as was shown in [4] for a different problem. Hence, if $\vec{\psi} \in C_{0}^{\infty}\left(G_{t_{0}}\right)$, then choosing $\phi \equiv 1$ on $\operatorname{supp}(\vec{\psi})$ yields

$$
\begin{aligned}
B_{2}(\vec{v}, \vec{\psi}) & =B_{2}(\phi \vec{v}, \vec{\psi})=\lim _{r \rightarrow \infty} B_{2}\left(\phi \vec{v}_{r}, \vec{\psi}\right) \\
& =\lim _{r \rightarrow \infty} \lambda_{r}\left(\phi \vec{v}_{r}, \vec{\psi}\right)=\lambda_{0}(\vec{v}, \psi) .
\end{aligned}
$$

Hence, $\vec{v} \geqslant 0$, $\neq \equiv \overrightarrow{0}$ satisfies $l_{2} \vec{v}=\lambda_{0} \vec{v}$, and, by the unique characterization of $\lambda$, $\lambda_{0}=\lambda\left(t_{0}\right)$. Since the limit is independent of the subsequence chosen, $\lim _{r \rightarrow \infty} \lambda_{r}=$ $\lambda\left(t_{0}\right)$ for the original sequence. To show that $\lambda(t) \rightarrow \infty$ as $t \rightarrow \infty$, observe that by the Gagliardo-Nirenberg estimates (see e.g. [6])

$$
B_{2}(\vec{\phi}, \vec{\phi}) \geqslant \frac{1}{2} \int \sum_{k, i}\left[D_{k}\left(\phi_{i}\right)\right]^{2}
$$

if $\vec{\phi} \in C_{0}^{\infty}\left(G_{t}\right), \operatorname{diam}\left(G_{t}\right)$ sufficiently small. We now apply Poincaré's Inequality [7] to each component. 
Applying Lemma 1 and Corollary 1 we find

COROLlary 3. If $\lambda\left(-\sqrt{b_{i j} b_{j i}}\right) \leqslant 0$ in $G_{t_{0}}$ there exists $G_{t} \subseteq G_{t_{0}}$ such that $\lambda\left(b_{i j}\right)=0$.

As an application we state the following result related to those given in $[5,1,2]$ for $n=1$, but not contained by them.

Corollary 4. Suppose $b_{i j} \leqslant a_{i j}$ in $G_{t_{0}}$ for all $i, j$ with $b_{i i}<a_{i i}$ for some $i$ and in some set of positive measure in $G_{t_{0}}$. Supporo further that $b_{i j}$ is symmetric and $a_{i j}+a_{j i} \leqslant 0, i \neq j$. If $B_{1}(\vec{u}, \vec{u}) \leqslant 0$ in $G_{t_{0}}, \vec{u} \neq \equiv \overrightarrow{0}$, then $l_{2}$ has eigenvalue 0 in some $G_{t}$ with $t>t_{0}$.

Proof. Since $b_{i j}$ is symmetric then the first condition implies $b_{i j} \leqslant \min \left(a_{i j}, a_{j i}\right)$ $\leqslant 0$. Hence, $b_{i j} b_{j i}=b_{i j}^{2} \geqslant\left[a_{i j}+a_{j i}\right]^{2} / 4$. The conclusion now follows from Corollaries 2(v) and 3.

Obviously, $b_{i i}(x)<a_{i i}(x)$ can be replaced by the other conditions of Corollary 2 . We also observe that the diagonal case shows that the assumption of the irreducibility of $B$ cannot be dropped.

We conclude by weakening condition (3). If $C$ is a constant invertible matrix in $G$ then $l_{3} \vec{u}=-\Delta \vec{u}+C^{-1} B C \vec{u}$ has the same spectrum as $l_{2}$. If $C^{-1} B C$ has nonnegative off-diagonal entries, then an analogue of the earlier results is valid. As an example we state

Corollary 5. Let $S\left(l_{2}\right)$ denote the spectrum of $l_{2}, m=2$. Assume that for some constant $\xi>0$ we have $b_{11}-b_{22}<-\left|b_{21} \xi-b_{12} \xi^{-1}\right|$ in $G$. Then

$$
S\left(l_{2}\right) \cap\left[\mu\left(\left(d_{i j}+d_{j i}\right) / 2\right), \mu\left(-\sqrt{d_{i j} d_{j i}}\right)\right] \neq \varnothing,
$$

where $C^{-1} B C=\left(d_{i j}\right)$ and $C=\left(\begin{array}{cc}\xi & \xi \\ -1 & 1\end{array}\right)$.

\section{REFERENCES}

1. S. Ahmad and A. C. Lazer, An N-dimensional extension of the Sturm separation and comparison theory to a class of nonselfadjoint systems, SIAM J. Math. Anal. 9 (1978), 1137-1150.

2. A new generalization of the Sturm comparison theorem to selfadjoint systems, Proc. Amer. Math. Soc. 68 (1978), 185-188.

3. W. Allegretto, A comparison theorem for nonlinear operators, Ann. Scuola Norm. Sup. Pisa 25 (1971), 41-46.

4. Positive solutions and spectral properties of second order elliptic operators, Pacific J. Math. 92 (1981), 15-25.

5. F. Dannan, Sturmian theory and disconjugacy of second order systems, Proc. Amer. Math. Soc. 90 (1984), 563-566.

6. A. Friedman, Partial differential equations, Holt, Rinehart and Winston, New York, 1969.

7. D. Gilbarg and N. S. Trudinger, Elliptic partial differential equations of second order, Springer-Verlag, Berlin, 1977.

8. M. A. Krasnoselskii, Positive solutions of operator equations, Noordhoff, Groningen, 1964.

9. K. Kreith, Oscillation theory, Lecture Notes in Math., vol. 324, Springer-Verlag, 1973.

10. O. Ladyzhenskaya and N. Uraltseva, Linear and quasilinear elliptic equations, Academic Press, New York, 1968.

11. M. H. Protter, The generalized spectrum of second order elliptic systems, Rocky Mountain J. Math. 9 (1979), 503-518. 
12. C. A. Swanson, Comparison and oscillation theory of linear differential equations, Academic Press, New York, 1968.

13. _ Picone's identity, Rend. Mat. 8 (1975), 373-397.

14. A dichotomy of PDE Sturmian theory, SIAM Rev. 20 (1978), 285-300.

15. R. Vyborny, Continuous dependence of eigenvalues on the domain, Lecture Ser. No. 42, Institute for Fluid Dynamics and Applied Mathematics, Univ. of Maryland, 1964.

Department of Mathematics, University of Alberta, Edmonton, Alberta, Canada T6G 2G1 